ethosuximide, and gabapentin. Anticonvulsants in addition to phenytoin with increased risk of erythema multiforme, Stevens-Johnson syndrome (SJS), and toxic epidermal necrolysis (TEN) include carbamazepine, oxcarbazepine, valproate, phenobarbital, and lamotrigine. The combination of carbamazepine and acetaminophen further increases the risk of hypersensitivity skin reactions [1]. Erythema multiforme as a synergistic side effect to a combination of phenytoin therapy and cranial radiotherapy is also reported [2]. The routine use of postoperative phenytoin as a prophylactic anticonvulsant in the absence of a history of seizures is discouraged [3][4].

The US FDA cautions that phenytoin or fosphenytoin should not be prescribed as an alternative to carbamazepine in patients who carry HLA-B*15.02, although the association with severe cutaneous reactions in Asians is weaker than that found with carbamazepine [5]. Current studies find that the HLA-DRB1*15.01 allele is a risk factor for AED-induced SJS/TEN among Han Chinese, whereas HLA-A*33.03, HLA-B*58.01, and HLA-DRB $1 * 03.01$ alleles may be protectors against AED-induced skin reactions, especially CBZ-SJS/TEN [6]. Patients of Han ethnicity living in northeastern China and having EPHX1 c.337T $>$ C polymorphisms also show an increased risk of developing CBZ-SJS/TEN, related to an increased concentration of a CBZ metabolite, CBZ-10,11epoxide [7]. The discovery of a functional link of genetic variants to the phenytoin, carbamazepine, and other aromatic AED-related hypersensitivity cutaneous reactions might lead to prospective preventive genetic testing.

\title{
References.
}

1. Gau SS, et al. J Clin Psychopharmacol. 2008 Oct;28(5):509-17.

2. Khafaga YM, et al. Acta Oncol. 1999;38(1):111-6.

3. Young B, et al. Child's Brain. 1983;10(3):185-92.

4. Rapp RP, et al. Neurosurgery. 1983 Sep;13(3):272-5.

5. Locharernkul C, et al. Epilepsia. 2008 Dec;49(12):2087-91.

6. Wang W, et al. Epilepsy Behav. 2014 Jun 17;37C:16-19.

7. He X-J, et al. Epilepsia. 2014 Aug;55(8):1301-6.

\section{VALPROATE-INDUCED REVERSIBLE BRAIN ATROPHY AND COGNITIVE DETERIORATION}

Pediatric neurologists at Dalhousie University, Halifax, Nova Scotia, report an 8year-old boy with rapid cognitive decline after a year-long course of valproate in varying dosages for myoclonic and atypical absence seizures. Sequential MRIs over a 1-year seizure free period revealed progressive brain atrophy and white matter signal changes. MR spectroscopy while taking valproate showed a normal lactate peak and a decreased $\mathrm{N}$-acetylaspartate to creatine ratio. Tests for mitochondrial or neurodegenerative diseases and liver transaminases were normal. POLG gene testing detected no pathogenic variant. After discontinuing valproate, MRI and MR Spectroscopy, and cognitive and school function returned to baseline. He is currently seizure-free while taking clobazam monotherapy. (Lovett M, Skidmore DL, Mohamed IS. Valproate-induced pseudoatrophy: Expanding the clinical and imaging spectrum. Pediatr Neurol 2014 Aug;51(2):284-5).

COMMENTARY. Four types of valproate-induced encephalopathy are described:

1) Direct toxic encephalopathy; 2) hyperammonemic encephalopathy; 3) hepatic 
encephalopathy; and 4) encephalopathy with cognitive deterioration and reversible cerebral atrophy. Brain pseudoatrophy and mental regression while taking valproate is reported in a child with a rare mitochondrial DNA mutation [1]. The current and 5 previously reported cases support a role of VPA in triggering a reversible dose-dependent mental deterioration with brain pseudoatrophy in children with epileptic seizures. Search for mtDNA mutation should be considered in a child treated with VPA who shows an unexplained clinical worsening and atrophic cerebral changes on MRI.

\section{References.}

1. Galimberti CA, et al. Neurology. 2006 Nov 14;67(9):1715-7.

\section{METABOLIC DISORDERS}

\section{5,10-METHYLENETETRAHYDROFOLATE REDUCTASE DEFICIENCY AND MYOCLONIC EPILEPSY}

Investigators from the Children's Hospital of Philadelphia, PA, and McGill University, Montreal, Quebec, CA, report an adolescent learning-disabled girl who presented at age 14 years with an epilepsy syndrome initially diagnosed as juvenile myoclonic epilepsy. Her seizures initially resolved while taking valproic acid but later became refractory. At age 15 years she became ataxic and developed leg weakness and cognitive decline. Withdrawal of VPA and substitution of lamotrigine did not halt the mental deterioration, and testing revealed elevated plasma homocysteine and decreased plasma methionine. The diagnosis of 5,10-methylenetetrahydrofolate reductase (MTHFR) deficiency was confirmed by reduced fibroblast MTHFR activity, and mutation analysis revealing 2 variants in the MTHFR gene and splice site and missense mutations. Therapy with folinic acid, betaine, and methionine produced improvements in muscle strength, less severe ataxia, decreased seizures, and improved EEG and EMG. (D'Aco KE, Bearden D, Watkins D, Hyland K, Rosenblatt DS, Ficicioglu C. Severe 5,10methylenetetrahydrofolate reductase deficiency and two MTHFR variants in an adolescent with progressive myoclonic epilepsy. Pediatr Neurol 2014 Aug;51:266-70).

COMMENTARY. MTHFR deficiency is an autosomal recessive inborn error of folate metabolism with defective remethylation of homocysteine to methionine. Elevated homocysteine and low methionine is a diagnostic marker for MTHFR deficiency. Infants typically present with seizures, microcephaly, neurological deterioration, and coma and death if untreated. Childhood presentation is milder, with developmental delay, and marfanoid appearance, but sometimes more severe with rapid neurological deterioration, seizures, and incoordination. MTHFR may also occur in adults, presenting as hereditary spastic paraplegia. Two unrelated families, each with 2 affected siblings, are reported from Hadassah Medical College, Jerusalem [1]. Treatment aims are normalization of plasma and CSF methionine levels and reduced homocysteinemia.

MTHFR deficiency should be considered in the differential diagnosis of progressive myoclonic epilepsy (PME). The most common causes of PME in a previously healthy adolescent are Unverricht-Lundborg disease, ME with ragged red fibers, neuronal ceroid lipofuscinosis, dentatorubro-pallidoluysian atrophy (DRPLA), and 